\title{
THE IMAGE OF THE AHLFORS FUNCTION
}

\author{
C. DAVID MINDA ${ }^{1}$
}

\begin{abstract}
ABSTRACr. Let $\Omega$ denote a maximal region on the Riemann sphere for bounded holomorphic functions and $p \in \Omega$. We present a class of examples to show that the complement in the unit disk of the image of the Ahlfors function for $\Omega$ and $p$ can be a fairly general discrete subset of the unit disk.
\end{abstract}

1. Introduction. Let $\Omega$ be a region on the Riemann sphere that supports nonconstant bounded holomorphic functions and let $p \in \Omega$. Set $\mathscr{B}=\{f: f$ is holomorphic in $\Omega$ and $f(\Omega) \subset B\}$, where $B=\{z:|z|<1\}$. The Ahlfors function for $\Omega$ and $p$ is the unique function $h$ in $\mathscr{B}$ such that

$$
h^{\prime}(p)=\max _{f \in \mathscr{D}} \operatorname{Re} f^{\prime}(p) .
$$

It is elementary to show that $h(p)=0$. This paper is concerned with the image, $h(\Omega)$, of the Ahlfors function.

First, we survey the known results. Ahlfors [1] showed that $h(\Omega)=B$ for regions $\Omega$ of finite connectivity that have no trivial boundary components. More precisely, he proved that $h$ expresses $\Omega$ as an $n$-sheeted branched covering of $B$, where $n$ is the order of connectivity of $\Omega$. In the general situation Havinson [5] and Fisher [2] demonstrated that $B \backslash h(\Omega)$ has analytic capacity zero; that is, every bounded holomorphic function defined on $h(\Omega)$ may be extended to a bounded holomorphic function on $B$. It is not difficult to give an example of a region $\Omega$ such that $B \backslash h(\Omega) \neq \varnothing$. For example, let $K$ be a closed subset of $B$ which has analytic capacity zero and $\Omega=B \backslash K$. If $0 \in \Omega$, then the Ahlfors function $h$ for $\Omega$ and 0 is the identity function, so $h(\Omega)=B \backslash K$. The question of the size of $B \backslash h(\Omega)$ becomes more interesting if it is required that $\Omega$ be a maximal region for bounded holomorphic functions in the sense of Rudin [11]. For such a maximal region $\Omega$, Fisher [3] raised the question of whether the Ahlfors function must map $\Omega$ onto $B$. Roding [9] answered this question in the negative by exhibiting a maximal region $\Omega$ and a point $p \in \Omega$ such that the Ahlfors function for $\Omega$ and $p$ omitted two values in $B$. We shall extend Roding's result by showing that an Ahlfors function for a maximal region can actually omit a fairly general discrete set of values in $B$.

2. The example. Suppose $K$ is a discrete subset of $B$ such that $K \cap \mathbf{R}=\varnothing$ and $\bar{K}=K$, where $\bar{K}$ denotes the reflection of the set $K$ in the real axis. Set $\Delta=B \backslash K$ and $\Delta^{+}=\Delta \cap H$, where $H=\{z: \operatorname{Im}(z)>0\}$. Observe that the open interval

Received by the editors January 11, 1981 and, in revised form, March 18, 1981.

AMS (MOS) subject classifications (1970). Primary 30A40, 30A42.

1 This research was completed while the author was visiting the University of California, San Diego during 1980-81. 
$(-1,1)$ is a free boundary arc of $\Delta^{+}$. Let $f: \bar{H} \rightarrow \Delta^{+}$be an analytic universal covering projection of the lower half-plane $\bar{H}$ onto $\Delta^{+}$; of course, $f$ is not uniquely determined. The function $f$ could be replaced by $f \circ T$, where $T$ is any conformal automorphism of $\bar{H}$. Let $\Gamma$ be the associated group of cover transformations; that is, $\Gamma$ is the group of all Möbius transformations $T$ which map $\bar{H}$ onto itself and satisfy $f \circ T=f$. Later, we shall need the fact that $\Gamma$ possesses a set of generators each of which is parabolic. Let us establish this result now. Fix some point $b \in \Delta^{+}$. Because $K \cap H$ is discrete, the fundamental group $\pi_{1}\left(\Delta^{+}, b\right)$ is generated by countably many Jordan loops $\gamma_{i}$ with the property that each $\gamma_{i}$ contains exactly one point of $K \cap H$ in its interior. Recall that $\Gamma$ and $\pi_{1}\left(\Delta^{+}, b\right)$ are isomorphic as follows. Fix a point $\tilde{b} \in \bar{H}$ with $f(\tilde{b})=b$. For $\gamma \in \pi_{1}\left(\Delta^{+}, b\right)$ let $\tilde{\gamma}$ be the unique lift of $\gamma$ having initial point $\tilde{b}$. The terminal point of $\tilde{\gamma}$ also lies over $b$ and there is a unique $T_{\gamma} \in \Gamma$ which sends $\tilde{b}$ to the terminal point of $\tilde{\gamma}$. The mapping $\gamma \rightarrow T_{\gamma}$ is an isomorphism. Since each $\gamma_{i}$ is retractable to an isolated boundary point, it follows that $T_{\gamma_{i}}$ is parabolic [7]. Thus, $\Gamma$ is generated by the $T_{\gamma_{i}}$, each of which is parabolic.

We use $f$ to construct another covering via the Schwarz reflection principle. Because $(-1,1)$ is a free boundary arc of $\Delta^{+}$, there is an open set $\sigma$ contained in the extended real line $R \cup\{\infty\}$ such that $f$ extends continuously to $\bar{H} \cup \sigma$ and $f$ maps each component of $\sigma$ homeomorphically onto $(-1,1)$. Without loss of generality, we may assume that $\infty \in \sigma$ and that $f(\infty)=0$. Let $\sigma_{\infty}$ be the component of $\sigma$ that contains $\infty$. Note that $\sigma$ is invariant under the group $\Gamma$; in fact, $\sigma=\cup T\left(\sigma_{\infty}\right)$, where the union is taken over all $T \in \Gamma$. We extend $f$ to a holomorphic function on $\Omega=\bar{H} \cup \sigma \cup H$ by means of the Schwarz reflection principle: $f(\bar{z})=\overline{f(z)}$. We continue to denote the extended holomorphic function by $f$. It is elementary to verify that $f: \Omega \rightarrow \Delta$ is an analytic covering, that $f^{\prime}(\infty)>0$ and that the group of cover transformations associated with this covering is exactly $\Gamma$.

\section{Proposition 1. $\Omega$ is a maximal region for bounded holomorphic functions.}

Proof. Suppose that $\Omega$ were not maximal. Let $\Omega^{*}$ be the unique maximal region on the Riemann sphere which contains $\Omega$ and has the property that every bounded holomorphic function on $\Omega$ extends to a bounded holomorphic function on $\Omega^{*}$. Obviously, any point of $\Omega^{*} \backslash \Omega$ lies on the real axis. Now, the covering projection $f$ itself extends to a holomorphic function $f^{*}: \Omega^{*} \rightarrow B$ and $f^{*}(\bar{z})=\overline{f^{*}(z)}$. Select $a \in \Omega^{*} \backslash \Omega$. Because $f^{*}$ is symmetric about the real axis, $f^{*}(a)=b \in(-1,1)$. Select $r>0$ so small that the closed disk $D=\{z:|z-b| \leqslant r\}$ is evenly covered by $f$. This means that $f^{-1}(D)=\cup D_{i}$, where $D_{i}$ is a compact subset of $\Omega, D_{i} \cap D_{j}=\varnothing$ if $i \neq j$ and $f \mid D_{i}$ is a homeomorphism of $D_{i}$ onto $D$. We claim that the sets $D_{i}$ cannot cluster at $a$. If they did, then we could find a sequence $\left(z_{n}\right)_{n=1}^{\infty}$ in $\Omega$ with $z_{n} \in \partial D_{i(n)}$ and $z_{n} \rightarrow a$. This would yield $f^{*}\left(z_{n}\right) \rightarrow b$, a contradiction, since $r=\left|f\left(z_{n}\right)-b\right|$ for all $n$. Hence, we can find an open neighborhood $V$ of $a$ in $\Omega^{*}$ such that $V \cap f^{-1}(D)=\varnothing$ and $f^{*}(V) \subset D$. Then $V \cap \Omega=\varnothing$ since $z \in V \cap \Omega$ implies $f^{*}(z) \in D$ which would give $z \in f^{-1}(D)$, a contradiction. Consequently, $V \subset$ $\Omega^{*} \backslash \Omega \subset \mathbf{R}$, which violates the fact that $V$ is open. Hence, $\Omega$ is maximal. 
LEMMA. Let $a x^{2}+b x+c$ be a real quadratic with distinct real roots $r$ and $s$, where $r<s$. Suppose $E_{0}$ is a measurable set whose closure is contained in $(r, s)$. Then

$$
\int_{E} \frac{d x}{a x^{2}+b x+c}=0
$$

where $E=\cup_{n \in \mathrm{Z}} E_{n}$ and $E_{n}=E_{0}+n(s-r)=\left\{x+n(s-r): x \in E_{0}\right\}$.

Proof. Without loss of generality, we may assume that $a=1$. Our first step is to show that we may specialize to the situation in which $r=-1$ and $s=1$. Let

$$
y=y(x)=\frac{2 x-r-s}{s-r}, \quad x=x(y)=\frac{1}{2}[(s-r) y+(s+r)] .
$$

Note that $y(r)=-1$ and $y(s)=1$. Then

$$
\int_{E_{n}} \frac{d x}{x^{2}+b x+c}=\int_{E_{n}} \frac{d x}{(x-r)(x-s)}=\frac{2}{s-r} \int_{F_{n}} \frac{d y}{y^{2}-1},
$$

where $F_{0}=\left\{y(x): x \in E_{0}\right\}$ and $F_{n}=F_{0}+2 n$. The closure of $F_{0}$ is contained in $(-1,1)$. Thus, it suffices to show that

$$
0=\int_{F} \frac{d y}{y^{2}-1}=\sum_{-\infty}^{\infty} \int_{F_{n}} \frac{d y}{y^{2}-1}
$$

where $F=\cup_{n \in \mathbf{Z}} F_{n}$.

Next, we establish (1) in the special case $F_{0}=(a, b)$, where $-1<a<b<1$. By direct calculation we obtain

$$
\begin{gathered}
\int_{F_{0}} \frac{d y}{y^{2}-1}=-\frac{1}{2} \log \left(\frac{1+b}{1-b} \cdot \frac{1-a}{1+a}\right) \\
\int_{F_{n}} \frac{d y}{y^{2}-1}=\log \left(\frac{b+2 n-1}{b+2 n+1} \cdot \frac{a+2 n+1}{a+2 n-1}\right), \quad n \neq 0 .
\end{gathered}
$$

Then

$$
\sum_{n=-N}^{N} \int_{F_{n}} \frac{d y}{y^{2}-1}=\frac{1}{2} \log \left(\frac{a+2 N+1}{a-2 N-1} \cdot \frac{b-2 N-1}{b+2 N+1}\right),
$$

which implies that (1) is valid in this special case.

Clearly, the preceding work implies that (1) also holds in case $F_{0}$ is a finite union of open intervals. Now, consider any measurable set $F_{0}$ whose closure is contained in $(-1,1)$. Let $\varepsilon>0$ be given. Select $u \in(0,1)$ so that the closure of $F_{0}$ is contained in $(-u, u)$. Next, determine $I_{0}$, a finite union of open intervals contained in $(-u, u)$, such that

$$
m\left(F_{0} \triangle I_{0}\right)<\eta=\varepsilon / \sum_{-\infty}^{\infty} \frac{1}{\left|(2|n|-u)^{2}-1\right|},
$$

where $F_{0} \triangle I_{0}$ denotes the symmetric difference of the sets $F_{0}$ and $I_{0}$ and $m$ denotes Lebesgue measure [10, p. 62]. Set $I_{n}=I_{0}+2 n$. Then

$$
\left|\sum_{-\infty}^{\infty} \int_{F_{n}} \frac{d y}{y^{2}-1}\right|=\left|\sum\left(\int_{F_{n}} \frac{d y}{y^{2}-1}-\int_{I_{n}} \frac{d y}{y^{2}-1}\right)\right| \leqslant \sum_{-\infty}^{\infty} \int_{F_{n} \Delta I_{n}} \frac{d y}{\left|y^{2}-1\right|} .
$$


Elementary estimates show that

$$
\int_{F_{n} \Delta I_{n}} \frac{d y}{\left|y^{2}-1\right|}<\frac{\eta}{\left|(2|n|-u)^{2}-1\right|}
$$

Thus,

$$
\left|\sum_{-\infty}^{\infty} \int_{F_{n}} \frac{d y}{y^{2}-1}\right| \leqslant \varepsilon
$$

so the proof is complete.

Proposition 2. If $h$ is the Ahlfors function for $\Omega$ and $\infty$, then $h \circ T=h$ for all $T \in \Gamma$.

Proof. In order to prove that $h \circ T=h$, it suffices to show that $(h \circ T)^{\prime}(\infty)=$ $h^{\prime}(\infty)$ since the Ahlfors function is unique. Let $E=\mathbf{R} \backslash \sigma$. Then $E$ is a compact subset of $\mathbf{R}$ and $T(E)=E$ for all $T \in \Gamma$. A result of Pommerenke [8] implies that

$$
h(z)=\frac{\exp (g(z))-1}{\exp (g(z))+1}
$$

where $g(z)=\frac{1}{2} \int_{E} d \zeta /(z-\zeta)$. Note that $g(\infty)=0$ and $g^{\prime}(\infty)=(1 / 2) m(E)$, where $m(E)$ denotes the Lebesgue measure of $E$ as a subset of $\mathbf{R}$. Consequently, $h^{\prime}(\infty)=\frac{1}{4} m(E)$ and

$$
(h \circ T)^{\prime}(\infty)=\frac{2 \exp (g \circ T(\infty))}{[\exp (g \circ T(\infty))+1]^{2}}(g \circ T)^{\prime}(\infty) .
$$

Clearly, it is enough to demonstrate that $g \circ T(\infty)=0$ and $(g \circ T)^{\prime}(\infty)=$ $(1 / 2) m(E)$.

First, we demonstrate that $(g \circ T)^{\prime}(\infty)=g^{\prime}(\infty)$. Suppose $T \in \Gamma$ and $T$ is not the identity, say $T(z)=(a z+b) /(c z+d)$, where $a, b, c, d \in \mathbf{R}$ and $a d-b c=1$. Note that $c \neq 0$; otherwise, $T$ fixes the point $\infty$ which implies that $T$ is the identity because the group $\Gamma$ is fixed point free on $\Omega$. Clearly, $T(\infty)=a / c$. Now, we calculate $(g \circ T)^{\prime}(\infty)=g^{\prime}(a / c) T^{\prime}(\infty)$. From the definition of $g$, we obtain

$$
g^{\prime}\left(\frac{a}{c}\right)=-\frac{1}{2} \int_{E} \frac{d \zeta}{(a / c-\zeta)^{2}} .
$$

We make the change of variable $\omega=T^{-1}(\zeta)=(d \zeta-b) /(-c \zeta+a)$ in this integral and obtain

$$
g^{\prime}\left(\frac{a}{c}\right)=-\frac{1}{2} \int_{E} c^{2} d \omega=-\frac{c^{2}}{2} m(E) .
$$

Here we have used the facts that $T(E)=E$ and $T$ preserves direction on $E$ because $T(H)=H$. From $T^{\prime}(\infty)=-1 / c^{2}$, we obtain $(g \circ T)^{\prime}(\infty)=\frac{1}{2} m(E)=$ $g^{\prime}(\infty)$.

Second, we demonstrate that $g \circ T(\infty)=0$ for any parabolic element $T$ of the group $\Gamma$. We assume that $T$ has the same form as earlier. The fact that $T$ is parabolic implies that $a+d= \pm 2$; without loss of generality we may assume that 
$a+d=2$. Then the unique fixed point of $T$ is $(a-1) / c \in E$. We want to show that

$$
g \circ T(\infty)=g\left(\frac{a}{c}\right)=\frac{1}{2} \int_{E} \frac{d \zeta}{a / c-\zeta}=0 .
$$

We shall make a change of variable in this integral which has the effect of converting $T$ into a translation. Define

$$
R(z)=\frac{1}{(a-1) / c-z}
$$

then $R^{-1}(z)=(a-1) / c-1 / z$. We make the change of variable $\zeta=R^{-1}(\omega)$ and obtain

$$
g \circ T(\infty)=\frac{c}{2} \int_{F} \frac{d \omega}{\omega(\omega+c)}
$$

where $F=R(E)$ is invariant under $U(z)=R \circ T \circ R^{-1}(z)=z-c$. Let $F_{0}$ be the part of $F$ that is contained in the open interval between $0=R(\infty)$ and $-c=$ $R(a / c) ; F_{0}$ is a compact subset of this open interval. Also, $F=\cup_{n \in \mathbf{Z}} F_{n}$, where $F_{n}=F_{0}+n c$, since $F$ is invariant under $U$. By applying the preceding lemma, we may conclude that $g \circ T(\infty)=0$.

The foregoing results imply that $h \circ T=h$ for any parabolic element $T$ of $\Gamma$. But, as we have already observed, $\Gamma$ is generated by parabolic elements. It now follows that $h \circ T=h$ for all $T \in \Gamma$.

Proposition 3. If $K$ is a discrete subset of $B$, then the analytic covering projection $f: \Omega \rightarrow \Delta$ is the Ahlfors function for $\Omega$ and $\infty$.

Proof. Let $h$ be the Ahlfors function for $\Omega$ and $\infty$. Then $h^{\prime}(\infty) \geqslant f^{\prime}(\infty)$. Proposition 2 shows that the function $h$ is invariant under the group $\Gamma$. This implies that $h$ induces an analytic function $\tilde{h}: \Delta \rightarrow B$ such that $h=\tilde{h} \circ f$. In fact, we may simply define $\tilde{h}(w)=h(z)$, where $z \in \Omega$ is any point such that $f(z)=w$. The function $\tilde{h}$ is well defined since $f\left(z_{1}\right)=f\left(z_{2}\right)$ if and only if there exists $T \in \Gamma$ with $z_{2}=T\left(z_{1}\right)$. Because the set $K$ is discrete, the Ahlfors function for $\Delta$ and the origin is the identity function. Hence, $\tilde{h}^{\prime}(0) \leqslant 1$, so that $h^{\prime}(\infty)=\tilde{h}^{\prime}(0) f^{\prime}(\infty)<f^{\prime}(\infty)$. This gives $f=h$ since the Ahlfors function is unique.

3. Summary and questions. Suppose that $K$ is a discrete subset of $B$ such that $\bar{K}=K$ and $K \cap \mathbf{R}=\varnothing$. Then the analytic covering $f: \Omega \rightarrow \Delta=B \backslash K$ that was constructed in the preceding section is the Ahlfors function for $\Omega$ and $\infty$. The region $\Omega$ is maximal for bounded holomorphic functions and $f(\Omega)=B \backslash K$. This is an improvement of the example of Roding [9] in which $K$ consisted of two points. Hence, the Ahlfors function for a maximal region can omit a discrete set of values. Recall that Havinson [5] and Fisher [2] have shown that the set of omitted values always has analytic capacity zero. Therefore, it is still an open question whether the Ahlfors function for a maximal region can actually omit an uncountable set of zero analytic capacity. Gamelin [4] has obtained some results on the range of the Ahlfors function. 
Other questions naturally suggest themselves. As usual, $\Omega$ denotes a maximal region for bounded holomorphic functions and $h$ an Ahlfors function for $\Omega$. We know that $h$ need not map $\Omega$ onto $B$. How does $\Omega$ cover $h(\Omega)$ ? For example, if $\Omega$ is infinitely connected, does each point of $h(\Omega)$ have infinitely many preimages? Also, does the function $h: \Omega \rightarrow h(\Omega)$ belong to $B l$ or $B l_{1}$ ? Here $B l$ and $B l_{1}$ are the classes introduced by Heins [6] and generalize the notions of inner function and Blaschke product to mappings between Riemann surfaces. In our example, the function $f$ has the property that each point of $f(\Omega)$ has infinitely many preimages and the function $f$ also belongs to the class $B l_{1}$ since it is a covering.

I would like to thank the referee for pointing out an error in the original version of the paper.

\section{REFERENCES}

1. L. V. Ahlfors, Bounded analytic functions, Duke Math. J. 14 (1947), 1-11.

2. S. D. Fisher, On Schwarz's lemma and inner functions, Trans. Amer. Math. Soc. 138 (1969), 229-240.

3.

4. T. W. Gamelin, Cluster values of bounded analytic functions, Trans. Amer. Math. Soc. 225 (1977), 295-306.

5. S. Ya. Havinson, Analytic capacity of sets, joint nontriviality of various classes of analytic functions and the Schwarz lemma in arbitrary domains, Amer. Math. Soc. Transl. (2) 43 (1964), 215-266.

6. M. Heins, On the Lindelöf principle, Ann. of Math. 61 (1953), 440-473.

7. A. Marden, I. Richards and B. Rodin, Analytic self-mappings of Riemann surfaces, J. Analyse Math. 18 (1967), 197-225.

8. Ch. Pommerenke, Über die analytische Kapazität, Arch. Math. (Basel) 11 (1960), 270-277.

9. E. Roding, Über die Wertannahme der Ahlfors funktion in beliebigen Gebieten, Manuscripta Math. 20 (1977), 133-140.

10. H. L. Royden, Real analysis, 2nd ed., Macmillan, New York, 1968.

11. W. Rudin, Some theorems on bounded analytic functions, Trans. Amer. Math. Soc. 78 (1955), 333-342.

Department of Mathematics, University of California, San Diego, la Jolla, California 92093

Current address: Department of Mathematical Sciences, University of Cincinnati, Cincinnati, Ohio 45221 